\title{
EAR-RT: Energy Aware Routing with Real-Time Guarantee for Wireless Sensor Networks ${ }^{\star}$
}

\author{
Junyoung Heo ${ }^{1}$, Sangho $\mathrm{Yi}^{1}$, Geunyoung Park $^{1}$, Yookun Cho ${ }^{1}$, \\ and Jiman Hong ${ }^{2, \star \star}$ \\ 1 Seoul National University \\ \{jyheo, shyi, gypark, cho\}@ssrnet.snu.ac.kr \\ 2 Kwangwoon University \\ gman@daisy.kw.ac.kr
}

\begin{abstract}
Most energy aware routing algorithms focus on the increasing the lifetime and long-term connectivity of the wireless sensor networks. But energy efficiency sacrifices the communication delay between source and sink node. Therefore, many researchers have mentioned of energy and delay trade-offs. But the delay was not the main concern. In this paper we propose EAR-RT, an real-time guaranteed routing protocol for wireless sensor networks without harming energy awareness. Simulation results show that our real-time routing algorithm provides real-time guaranteed delivery while network is stable.
\end{abstract}

\section{Introduction}

Wireless sensor networks typically consist of hundreds or thousands of sensor nodes deployed in a geographical region to sense events. Wireless sensor networks provide a high-level description of the events being sensed. They are used in many applications such as environmental control, civil engineering, automatic manufacturing, habitant monitoring and so on. They can be used even in harsh environment [1,2]. Therefore, their developing entails significant technical challenges due to the many environmental constraints.

There are many research issues in the area of sensor networks because of existing a lot of constraints of sensor nodes. The energy constraints is the most important one of them. Sensor nodes are supplied with power generally by builtin battery. In addition, transmission of a packet in wireless sensor networks is expensive. Therefore, routing, which is highly correlated with energy consumption, is a critical factor determining the performance of network.

Most previous researches on routing [3, 4, 5, focused on the algorithm design and performance evaluation in terms of the packet transmission overhead

\footnotetext{
* The present research was conducted by the Research Grant of Kwangwoon University in 2006, and was supported in part by the Brain Korea 21 Project.

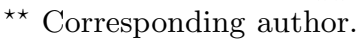


and loss rate. On the other hand, some routing algorithms were proposed in $[1,2,6,7,8,8$ in order to improve scalability of routing algorithms for large sensor networks.

In our proposed algorithm, the energy drain rate and the residual energy of each sensor node are used as the routing information, updated per almost every communication with energy awareness. To obtain such information, our algorithm takes advantage of the characteristic of wireless network, called overhearing. When two nodes communicate with each other, the neighboring nodes can overhear the packet being transmitted. Overhearing enables each sensor node to obtain the energy information of neighboring nodes without additional overhead. Then, they update their routing tables using this information. Therefore, our algorithm can provide longer connectivity of sensor networks through efficient use of energy among the nodes in the network. In addition, our algorithm considers real-time communication. Real-time packets must be arrived at sink node before deadline. But existing energy-aware routing delayed packets to save energy. So, these routing algorithms are not suitable for real-time packet transmission.

The rest of the paper is organized as follows. In Section 2 we present related works. Section 3 describes a new energy aware routing algorithm with realtime property. Section 4 presents and evaluates the performance of the proposed algorithm against the prior algorithm. Finally, some conclusions are given in Section 5.

\section{Related Works}

There are a lot of research results on sensor network routing for energy awareness and real-time communications.

In [3], Ganesan et al. proposed the use of braided multi-paths instead of completely disjoint multi-paths in order to keep the cost of maintaining the multi-paths low. The costs of such alternate paths are also comparable to the primary path because they tend to be much closer to the primary path.

In [4, Chang and Tassiulas proposed an algorithm for maximizing the lifetime of a network by selecting a path whose nodes have the largest residual energy. In this way, the nodes in the primary path retain their energy resources, and thus avoid having to continuously rely on the same route. This contributes to ensuring longer life of a network.

In [7, Chang and Tassiulas also applied this combined metric concept for direct routing. Their algorithm is proposed to maximize the lifetime of a network when data rate is known. The main idea is to avoid using the nodes with low energy and to choose the shortest path.

In [5], Li et al. proposed an algorithm in which the constraint of the residual energy of a route is relaxed slightly to select a more energy efficient route. It is generally known that a route with the largest residual energy for routing a packet entails high energy consumption. Therefore, there is a trade-off between minimizing the total consumed energy and the residual energy of the network. In that paper, groups of the sensors in geographic proximity were clustered 
together into a zone and each zone was treated as a separate entity and allowed to determine how it will route a packet across.

$\mathrm{Lu}$ et al. proposed real-time communication architecture for sensor networks [9]. They proposed velocity monotonic scheduling for packet scheduling on a node. They did not consider end-to-end delay between source and sink.

He et al. proposed end-to-end real-time communication protocol, SPEED [10]. SPEED maintains a desired delivery speed through a combination of feedback control and non-deterministic geographic forwarding. By doing so, it achieved real-time communication. However it did not consider energy awareness.

\section{EAR-RT: Energy Aware Routing with Real-Time Guarantee}

We had proposed energy aware routing with dynamic probability scaling (EARDPS) in our previous work[11]. This work is based on the energy aware routing (EAR) proposed by Shah and Rabaey 1]. EAR uses maintenance phase to update residual energy of sensor nodes. But EAR-DPS uses overheard packets without maintenance phase to update residual energy. Our proposed algorithm to support real-time communication is based on EAR-DPS.

EAR and EAR-DPS find multiple routes, if any, from source to destination nodes. Each route is assigned a probability of being selected to transmit a packet, based on residual energy and the energy for communications at the nodes along the route. Then, based on these probabilities, one of the candidate routes is chosen in order to transmit a packet. The probability is proportional to the energy level at each node, so the routes with higher energy are more likely to be selected. EAR protects any route from being selected all the time, preventing the energy depletion [1,11]. However, the end-to-end delay of communication may increase. So, EAR and EAR-DPS cannot be used for real-time communication. EAR-RT resolves this problem by considering the deadline of packets.

The operation of EAR-RT consists of two phases: setup phase and data communication phase. Basically EAR and EAR-DP use similar process. In setup phase, the sink node initiates a route request and a routing table is built up by finding all the paths from a source to the sink and their energy cost and time delay. In data communication phase, data packets are sent from the source to the sink. Each intermediate node forwards the packet to a neighboring node, which is chosen randomly among neighboring nodes that can deliver the packet in time. This probability is inversely proportional to the energy cost of neighboring nodes.

Before describing our EAR-RT algorithm, we make a list of some common notations used in this paper in Table 1.

\subsection{Setup Phase}

The sink node initiates the setup phase by flooding a route request message. A route request message has energy $\operatorname{cost}\left(\right.$ Cost $\left._{i}\right)$, time delay $\left(\right.$ Time $\left._{i}\right)$ and residual $\operatorname{energy}\left(R_{i}\right)$ of a node. Cost $_{\text {sink }}$, energy cost of the sink node is 0 . Time $_{\text {sink }}$ is also 0 . 
Table 1. List of notations used in this paper

\begin{tabular}{|c|l|}
\hline$C_{i, j}$ & $\begin{array}{l}\text { Expected energy cost to send a packet from node } i \text { to the sink node via } \\
\text { node } j .\end{array}$ \\
\hline Cost $_{i}$ & Expected energy cost to send a packet from node $i$ to the sink node. \\
\hline$E_{i, j}$ & Energy cost to send a packet from node $i$ to node $j$ directly. \\
\hline$T_{i, j}$ & $\begin{array}{l}\text { Expected time delay to send a packet from node } i \text { to the sink node via } \\
\text { node } j .\end{array}$ \\
\hline Time $_{i}$ & Expected time to send a packet from node $i$ to the sink node. \\
\hline$H_{i, j}$ & $\begin{array}{l}\text { Time to send a packet from node } i \text { to node } j \text { directly. It includes trans- } \\
\text { mission delay, queuing delay and so on. }\end{array}$ \\
\hline$P_{i, j}$ & Probability to select node $j$ to forward a packet at node $i$. \\
\hline
\end{tabular}

When a node receives a route request message from neighboring nodes, it adds neighboring nodes only which are closer to sink node than itself into routing table. Then it calculates its energy cost and time delay. Then it forwards a route request message with its energy cost and time delay.

If node $i$ receives a route request message from node $j$ which is closer to sink node than node $i$, then

$$
\begin{aligned}
\text { Cost }_{i} & =\sum_{j \in R T} P_{i, j} C_{i, j} \\
\text { Time }_{i} & =\sum_{j \in R T} P_{i, j} T_{i, j} \\
C_{i, j} & =\text { Cost }_{j}+E_{i, j} \\
T_{i, j} & =\text { Time }_{j}+H_{i, j} \\
P_{i, j} & =\frac{1 / C_{i, j}}{\sum_{k \in R T} 1 / C_{k, i}} \\
E_{i, j} & =\left(d_{i j}^{3}\right)^{\alpha} / R_{j}^{\beta},
\end{aligned}
$$

where RT is routing table, $d_{i j}$ is the distance between node $i$ and node $j, R_{j}$ is the residual energy at node $j$ normalized to its initial energy, $\alpha$ and $\beta$ are weighting factors 1,11 .

The $R_{i}$, residual energy decreases as time goes on. So the residual energy should be informed to neighbor nodes frequently. EAR-DPS and EAR-RT make use of overhearing packets to update the residual energy. In the wireless networks, when two nodes communicate with each other, the neighboring nodes of a sender can hear the packet being transmitted.

\subsection{Data Communication Phase}

After setup phase is completed, each sensor node sends data packets that are collected to the sink node. We consider only a real-time packet with deadline. The deadline may be predefined or determined by nodes at every transmission. The laxity is embedded in a packet and re-calculated at every node on a path to the sink node. 
The path to the sink node is built up while transmission of a packet. Every node - including source node - selects the next node according to the following rules:

1. Select nodes in routing table which can deliver a packet within deadline.

$$
R T^{\prime}=\left\{j \mid T_{i, j} \leq \text { laxity_of_a_packet }\right\}
$$

2. Calculate probability based on the $R T^{\prime}$

$$
P_{i, j}^{\prime}=\frac{1 / C_{i, j}}{\sum_{k \in R T^{\prime}} 1 / C_{k, i}}
$$

3. Select the next node randomly by the probability, $P^{\prime}$.

4. When a node $i$ selects node $j$ as the next hop, node $i$ estimates the energy needed to transmit a data packet to node $j$ and its residual energy after transmitting.

5. Node $i$ forwards the data packet including its residual energy $R_{i}$, current cost Cost $_{i}$, Time $_{i}$ and new laxity that decreases by $H_{i, j}$.

6. Every node $k$ that overhear the packet and its routing table has the node $i$ recomputes $C_{k, i}$ based on $R_{i}$ and Cost $_{i}$ in the packet. Also $T_{k, i}$ is recomputed based on Time $_{i}$. Then it recomputes the probability $P$, Cost $_{k}$ and Time $_{k}$ based on new $C_{k, i}$ and $T_{i, i}$. At this moment, the residual energy of node $i$ is reflected in its neighbors' routing table.

7. This process continues until the data packet reaches the sink node.

\section{Experimentation}

We ran a simulation to evaluate our EAR-RT. In this simulation, the area of the sensor networks was assumed to be $100 \mathrm{~m} \times 100 \mathrm{~m}$. The number of the nodes in the network was assumed to be 100 nodes - one node is sink node while the others are sensor nodes. The sink node was located at the center of the field and the sensor nodes are placed randomly in the field. All of the sensor nodes sent data to the sink node at fixed interval. The length of interval between transmissions at each node was all identical. This interval can be considered as a virtual time unit. That is, all of the sensor nodes send their data to the sink node just once a single time unit. Every packet from sensor nodes has a deadline. The deadline is randomly chosen between 5 and 8 hops.

Every node was given an identical amount, 0.3J, of initial energy. Energy for transmission was assumed $20 \mathrm{~nJ} / \mathrm{bit}+1 \mathrm{pJ} / \mathrm{bit} / \mathrm{m}^{3}$. Energy for reception was assumed 30nJ/bit 1,11. The packet length was assumed 256 bits. Energy metric function with $\alpha=1$ and $\beta=50$ was used. In this simulation, the following assumptions were made:

- Every node knows its position and distance between itself and other nodes.

- Every node has identical maximum radio range, $20 \mathrm{~m}$.

Figure 1 shows the residual energy of each node at time 600 of a network using EAR-RT and EAR-DPS. Each rectangle indicates sensor node and number in a 

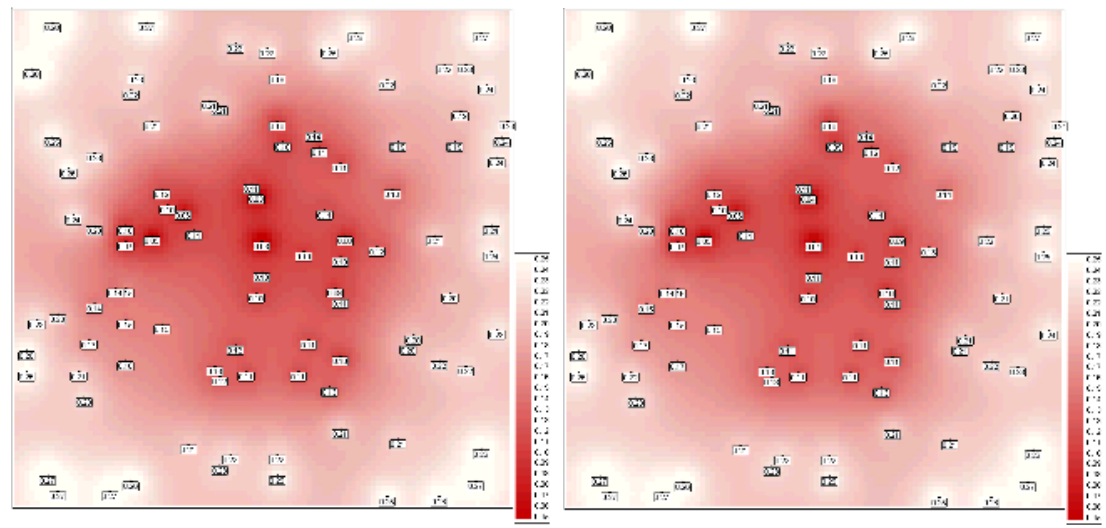

Fig. 1. (a) Residual energy of EAR-RT (b) Residual energy of EAR-DPS
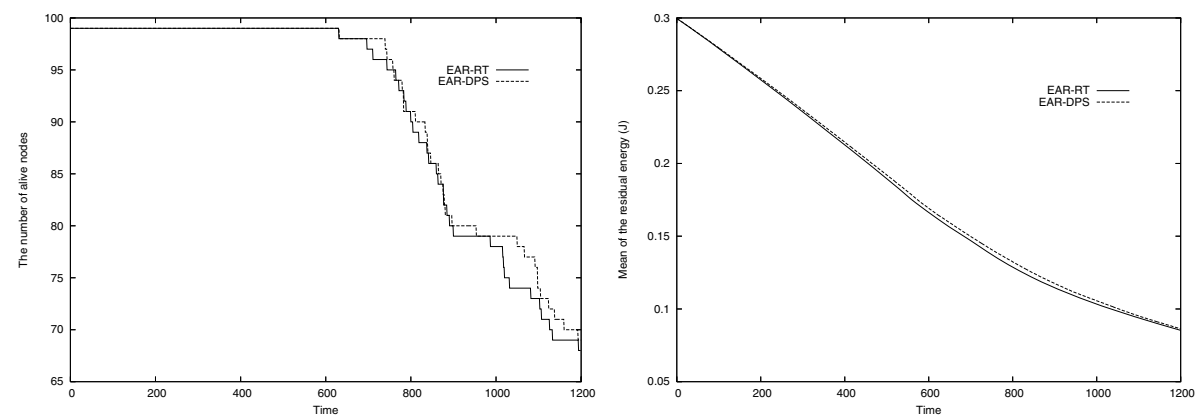

Fig. 2. (a) The number of active nodes during communication phase (b) Mean of residual energy of each node

rectangle indicates residual energy of a node. Nodes in dark area have smaller energy than those in light area. This figure shows that both algorithms affect the residual energy of nodes alike.

Figure 2(a) shows the number of active nodes in the network. Figure 2(b) shows the average of residual energy of each node. These figures tell us that the energy awareness of EAR-RT is not inferior to EAR-DPS. In case of EAR-RT, the number of alive nodes and average residual energy are slightly less than EAR-DPS. However EAR-RT improved the real-time property by sacrificing its energy awareness a little.

Figure 3 shows the deadline miss ratio of EAR-RT and EAR-DPS. EARRT delivered more packets in time compared to EAR-DPS. While the network is stable, EAR-RT delivered most packets in time. But some packets of EARDPS cannot meet the deadline because considering only energy. From these results, our EAR-RT is suitable for real-time communications while keeping energy awareness in sensor networks. 


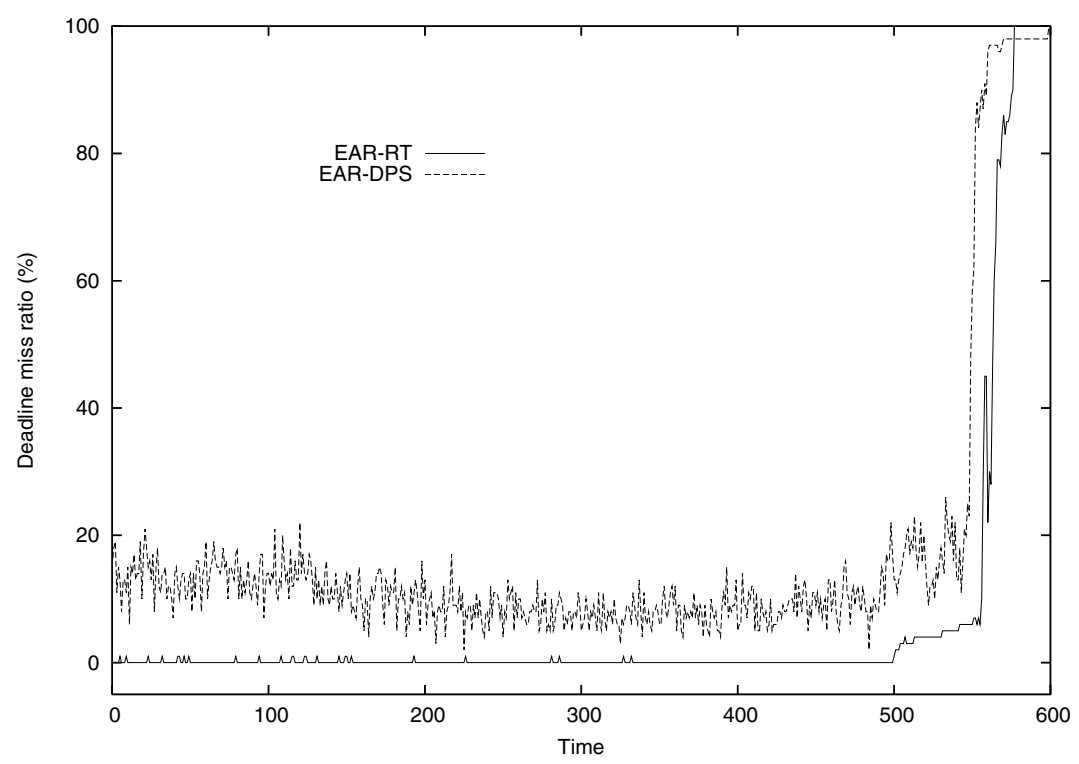

Fig. 3. Deadline miss ratio

In Figure 3, around of time 550, the miss ratio increases rapidly. At that time, nodes around sink node were dead and the network was partitioned. So, most packets could not be delivered to the sink node.

\section{Conclusion}

We proposed EAR-RT, an energy aware routing algorithm with real-time guarantee for high survivability and real-time communication of wireless sensor networks. Our algorithm provide real-time communication without harming energy awareness of existing energy aware routing algorithm, EAR-DPS. Simulation results showed that our algorithm reduced deadline miss ratio compared to existing algorithm. Consequently our algorithm can provide longer connectivity of sensor networks and ensure efficient use of energy among the nodes in the sensor network with guaranteeing real-time properties.

\section{References}

1. Shah, R., Rabaey, J.: Energy aware routing for low energy ad hoc sensor networks. In: IEEE Wireless Communications and Networking Conference (WCNC), Orlando, FL. (2002)

2. Heinzelman, W.R., Chandrakasan, A., Balakrishnan, H.: Energy-efficient communication protocol for wireless microsensor networks. In: HICSS '00: Proceedings of the 33rd Hawaii International Conference on System Sciences-Volume 8, Washington, DC, USA, IEEE Computer Society (2000) 8020 
3. Ganesan, D., Govindan, R., Shenker, S., Estrin, D.: Highly-resilient, energyefficient multipath routing in wireless sensor networks. SIGMOBILE Mob. Comput. Commun. Rev. 5 (2001) 11-25

4. Chang, J.H., Tassiulas, L.: Maximum lifetime routing in wireless sensor networks. IEEE/ACM Trans. Netw. 12 (2004) 609-619

5. Li, Q., Aslam, J., Rus, D.: Hierarchical power-aware routing in sensor networks. In: DIMACS Workshop on Pervasive Networking. (2001)

6. Schurgers, C., Srivastava, M.B.: Energy efficient routing in wireless sensor networks. In: IEEE Military Communications Conference (MILCOM). (2001) 357361

7. Chang, J.H., Tassiulas, L.: Energy conserving routing in wireless ad-hoc networks. In: INFOCOM (1). (2000) 22-31

8. Braginsky, D., Estrin, D.: Rumor routing algorthim for sensor networks. In: WSNA '02: Proceedings of the 1st ACM international workshop on Wireless sensor networks and applications, New York, NY, USA, ACM Press (2002) 22-31

9. Lu, C., Blum, B.M., Abdelzaher, T.F., Stankovic, J.A., He, T.: Rap: A real-time communication architecture for large-scale wireless sensor networks. Technical report, Charlottesville, VA, USA (2002)

10. He, T., Stankovic, J.A., Lu, C., Abdelzaher, T.: Speed: A stateless protocol for real-time communication in sensor networks. In: ICDCS '03: Proceedings of the 23rd International Conference on Distributed Computing Systems, Washington, DC, USA, IEEE Computer Society (2003) 46

11. Park, G., Yi, S., Heo, J., Choi, W.C., Jeon, G., Cho, Y., Shim, C.: Energy aware routing with dynamic probability scaling. Lecture Notes in Computer Science 3642 (2005) 662-670 